\title{
Paternalismo industrial, empresa extranjera y campamentos mineros en América Latina: un esfuerzo de historia laboral y transnacional
}

\author{
Ángela Vergara \\ California State University \\ Los Angeles (USA) \\ avergar@exchange.calstatela.edu
}

\begin{abstract}
Resumen
El presente trabajo analiza las prácticas paternalistas desarrolladas por los empresarios industriales y mineros latinoamericanos durante la primera mitad del siglo XX. Desde la perspectiva de la historia del trabajo y de los recientes debates sobre lo transnacional, este artículo busca reflexionar en torno a los elementos comunes del paternalismo industrial a lo largo del continente así como su impacto en la configuración de las relaciones laborales. A partir de este análisis, se plantea la importancia que tuvo el paternalismo como ideología patronal y símbolo de modernidad y progreso que buscaba crear una mano de obra eficiente y productiva. A pesar de los amplios beneficios sociales y de la construcción de modernas villas obreras y campamentos mineros, el paternalismo industrial en América Latina no desplazó las tradiciones más represivas del empresariado ni logró controlar a los trabajadores.
\end{abstract}

Palabras clave: Paternalismo industrial, Campamento minero, Historia trasnacional, Empresarios

\begin{abstract}
This article analyzes the history of paternalism and welfare capitalism in Latin American mines and industries during the first half of the twentieth century. From the perspective of labor history and the recent debates about transnational history, this article examines the similarities among different experiences of welfare capitalism as well as its impact on labor relations. Building on this analysis, it argues the importance of welfare capitalism as a managerial ideology and symbol of modernity and progress that sought to create an efficient and productive labor force. Despite the wide range of social benefits and the building of modern working-class neighborhoods and mining camps, Latin American welfare capitalism never displaced repressive practices and, as a result, was unable to shape the labor force.
\end{abstract}

Key Words: Welfare capitalism, Mining camps, Trasnational history, Management.

VERGARA, Ángela, "Paternalismo industrial, empresa extranjera y campamentos mineros en América Latina: un esfuerzo de historia laboral y transnacional", en Avances del Cesor, Año X, N 10, 2013, pp. 113-128. 


\section{Introducción}

Desde fines del siglo XIX los esfuerzos por crear y asentar una mano de obra moderna estuvieron en el centro de los debates sobre la expansión del sector exportador e industrial en América Latina. Sin dejar nunca de lado la coerción, tanto industriales de diversa índole como empresarios mineros y ferroviarios fueron incluyendo una variada gama de beneficios sociales de corte paternalista. La construcción de barrios obreros, campamentos mineros y ciudades empresas (company towns) fueron parte de este esfuerzo. A través de la implementación de un complejo sistema paternalista se buscaba crear sistemas más eficientes de reclutamiento laboral, disminuir el ausentismo, reforzar vínculos de lealtad, capacitar a los trabajadores y, en última instancia, aumentar la productividad. Estas prácticas jugaron un papel esencial en la configuración de las relaciones e identidades sociales, laborales y políticas de importantes e influyentes sectores de la clase obrera latinoamericana. Sí por un lado las experiencias fueron altamente diversas, notándose fuertes diferencias en cuanto a la ubicación geográfica de los centros productivos (nivel de aislamiento), tipo y organización productiva, origen de la mano de obra (etnicidad y nacionalidad), relación con el Estado y gobiernos locales/municipales y características del empresariado (nacional o extranjero). Por otro lado, es posible establecer una suerte de "modelo" o tipología que se caracterizó por el esfuerzo de utilizar (y manipular) la entrega de beneficios sociales y servicios urbanos (vivienda, escuela, salud, etc.) con el fin de crear una mano de obra estable, dócil y productiva, estableciendo una relación de fuerte dependencia de los trabajadores y sus familias hacia las empresas ${ }^{1}$.

En este artículo buscamos reflexionar en torno a la historia del paternalismo industrial como elemento esencial de un modelo de producción industrial y gestión empresarial que ejerció una profunda influencia en América Latina durante la primera mitad del siglo XX. Este modelo se ve con especial claridad en los cambios que afectaron a la minería y la configuración de los nuevos campamentos mineros a partir de comienzos del siglo XX. Desde una perspectiva más amplia, este artículo plantea la importancia de entender el paternalismo industrial, las ciudades empresas y los campamentos mineros desde una perspectiva transnacional, lo cual posibilita contextualizar y comparar una variedad de experiencias locales a lo largo del continente como así tambien entender históricamente los intercambios y flujos de ideas y experiencias. Sin desconocer la relevancia, riqueza y singularidad de las experiencias locales, este esfuerzo comparativo nos ofrece la oportunidad de reconstruir la circulación de ciertas ideas y modelos de producción a lo largo de las Américas. A partir de

1 Los casos más completos de paternalismo industrial se dieron en las llamadas ciudades empresas o company towns. Como ejemplo véase los trabajos que se incluyen en: BORGES, Marcelo y TORRES, Susana (compiladores), Company Towns: Labor, Space, and Power Relations Across Time and Continents, Palgrave McMillian, Nueva York, 2012; DINIUS, Oliver y VERGARA, Ángela (coordinadores) Company Towns in the Americas, Georgia University Press, Athens, Georgia, 2011.

\section{4}


este esfuerzo comparativo, se busca contribuir a pensar y escribir una historia del trabajo que sin olvidar lo local pueda insertarse en el debate de lo transnacional o, como algunos historiadores del trabajo han señalado, en el debate de lo trans-local.

\section{Una historia transnacional del trabajo}

En los últimos años ha surgido un nuevo interés por entender o expandir el enfoque histórico desde lo estrictamente local/nacional hacia lo transnacional o a lo que va más allá de las fronteras del Estado nación ${ }^{2}$. Sin lugar a dudas, la profundización de los procesos de globalización y sus enormes consecuencias sociales, culturales, políticas y económicas a nivel local han levantado nuevas preguntas al campo de la historia y cuestionado las formas tradicionales de hacer y pensar la historia. De esta forma, los historiadores se han comenzado a preocupar, entre otras cosas, por estudiar los cambios en las fronteras, los intercambios y la mobilidad de personas e ideas a través del tiempo. Aunque en América Latina, tal como lo señala la historiadora Aviva Chomsky, estos debates no son completamente nuevos y se remotan a los estudios sobre el colonialismo, las relaciones centro-periferia y la dependencia, la discusión actual se ha re-enfocado más bien en el tema de los encuentros y conflictos más allá del espacio nacional o de las relaciones entre los estados ${ }^{3}$.

Si el debate sobre lo transnacional ha redefinido sobre todo los estudios culturales y la historia de las ideas, su impacto sobre las formas de hacer y pensar la historia del trabajo ha sido bastante menor - con la notable excepción- de los estudios sobre esclavitud que han estado tradicionalmente insertos en el debate sobre el mundo Atlántico ${ }^{4}$. En general, los historiadores laborales lationamericanos (sobre todo aquellos influidos por la llamada

2 Véase por ejemplo: COOPER, Frederick "What Is the Concept of Globalization Good For? An African Historian's Perspective", en African Affairs, Oxford University Press, 2001, № 100, pp. 189213. SHUKLA, Sandhya y TINSMAN, Heidi (coordinadoras) Imagining Our Americas: Toward a Transnational Frame, Duke University Press, Durham, 2007. SIEGEL, Micol, "Beyond Compare: Comparative Method after the Transnational Turn", en Radical History Review, invierno 2005, N ${ }^{\circ}$ 91, pp. 62-90.

3 Aviva Chomsky resalta la enorme influencia de los trabajos de Franz Fanon, Edward Said y C.L.R James, quienes a partir del estudio del colonialismo y anti-colonialismo comenzaron a explorar la relación entre distintas partes del mundo. CHOMSKY, Aviva, "Labor History as World History: Linking Regions Over Time”, en FINK, Leon (coordinador) Workers across the Americas: The Transnational Turn in Labor History, Oxford University Press, New York, 2011, pp. 23-32.

4 La literatura sobre el mundo Atlántico es extensa. Cabe destacar los influyentes trabajos de Sidney Mintz, especialmente Dulzura y poder: El lugar del azúcar en la historia moderna, Editorial Siglo XXI, México, 1996. Un trabajo más reciente es el de REIS, João José, DOS SANTOS GOMES, Flávio y DE CARVALHO, Marcus Joaquim, O Alufá Rufino: tráfico, escravidão e liberdade no Atlântico negro (1822-1853), Companhia das Letras, São Paulo, 2010. 
nueva historia del trabajo y los trabajos de Edward P. Thompson) se han concentrado en estudiar lo local. A partir de los estudios de fábricas, centros productivos, instituciones o grupos específicos de trabajadores, han reconstruido el mundo obrero y popular desde abajo, contribuyendo así a entender el complejo entramado de las relaciones laborales, sociales y políticas de la clase trabajadora. El gran desafío de la historia laboral ha estado en recuperar lo local y en contar la historia desde abajo y desde la perspectiva de sus propios protagonistas. Asimismo en los últimos años, sobre todo en el caso de Brasil y Argentina, se ha consolidado una historia regional del trabajo, que ha enfatizado la multiplicidad de experiencia dentro de los distintos países como así también cuestionado las visiones excesivamente centralizadoras y nacionales. Este fuerte regionalismo que ha marcado la historia laboral latinoamericana ha dificultado los intercambios y colaboraciones, encerrándonos en temáticas que muchas veces resultan difícil comparar. ${ }^{5}$

Sin embargo, a la luz de los nuevos debates sobre transnacionalidad, resulta importante buscar la forma de comprender e insertar lo local dentro de debates más amplios, que nos permitan establecer comparaciones, entender grandes procesos políticos y económicos. Igualmente las formas en la cual los procesos de globalización han impactado -muchas veces de forma desigual- las condiciones de vida y las formas y experiencias de trabajo y de organización de los trabajadores requiere con suma urgencia un análisis histórico. Asi, tal como lo señala Aviva Chomsky, incorporar lo transnacional nos permite entender cómo opera el sistema capitalista, cómo éste se articula con prácticas tradicionales y de subsistencia y cómo cambian y se adaptan los sistemas de trabajo locales a las demandas del capitalismo global ${ }^{6}$. La reciente recopilación de artículos de historia del trabajo realizadas por el historiador norteamericano Leon Fink sugiere nuevas temáticas y metodologías para comprender y estudiar lo laboral desde una perspectiva transnacional7. Definiendo "las Américas" como espacio de encuentros y conflictos y sin desconocer la profunda desigualdad entre ambos extremos del continente, los distintos artículos contenidos en dicho libro apuntan la importancia de lo transnacional para entender la historia del trabajo y de los trabajadores.

En esta línea se insertan los recientes esfuerzos por re-examinar las migraciones laborales,

5 Algunas de estas ideas son frutos de las reflexiones a partir de la rica discusión que se dio en la mesa redonda "A América Latina e a história social do trabalho numa perspectiva global" en el marco del II Seminário Internacional Mundos do Trabalho, Rio de Janeiro, Brasil, 27-30 noviembre de 2012. Integraron la mesa redonda: Rossana Barragán, Mirta Lobato, Carlos Ilades y Ángela Vergara.

6 CHOMSKY, Aviva, "Labor History ..." Op. Cit., y "Poblaciones a través de fronteras soberanas: raza, trabajo, migración y soberanía en el mundo contemporáneo" en Nostromo: Revista Crítica Latinoamericana, primavera-verano 2010, V. III, No III. En su libro sobre la industria textil en Colombia y Nueva Inglaterra, Chomsky demuestra la importancia de estas propuestas a través de un caso de estudio, CHOMSKY, Aviva, Linked Labor Histories: New England, Colombia, and the Making of a Global Working Class, Duke University Press, Durham, 2008.

7 FINK, Leon, Workers..., Op. Cit. 
la circulación de ideas políticas y sindicales, los espacios trans-fronterizos, la solidaridad internacional o la influencia del capital extranjero ${ }^{8}$. En el caso de la región Patagónica, por ejemplo, historiadores como Alberto Harambour y Ernesto Boholavsky han estudiado la circulación de bienes, ideas y personas en este espacio austral, demostrando los vínculos y contactos que existían entre ambos lados de la frontera, una frontera, que como Bohalavsky claramente señala, era articulada y actuaba como un hinterland ${ }^{9}$. En forma similar, la frontera Mexico-Estados Unidos ha comenzado a ser analizada como un espacio de trabajo transnacional, marcado no sólo por el flujo de personas sino también de productos, bienes de capital e ideas. Tal como lo señalan los estudios sobre el sistema bracero, esta frontera y sus influencias se extendían más allá de los estados limítrofes ${ }^{10}$. Igualmente, nuevos estudios históricos han comenzado a reexaminar la experiencia de trabajo y vida en las empresas de propiedad extranjera, señalando, por ejemplo, la enorme influencia de ideas de superioridad racial y cultural que imponían los empresarios y técnicos norteamericanos en lugares como el canal de Panamá, los campamentos de Petróleo en Maracaibo o las bases militares en el Caribe ${ }^{11}$.

Tomando lo transnacional como eje central, las experiencias de paternalismo industrial en América Latina nos ofrecen la oportunidad de examinar una serie de temas laborales que claramente trascienden lo estrictamente local. En términos más específicos buscamos comprender como los procesos de industrialización y modernización requirieron nuevas prácticas empresariales y hasta que punto existen semejanzas o es posible identificar una

8 Véase a modo de ejemplo: LIDA, Clara e ILADES, Carlos, "El anarquismo Europeo y sus primeras influencias en México después de la Comuna de Paris, 1871-1881”, en Historia Mexicana, 2001, L: 1, pp. 103-149.

9 BOHOLAVSKY, Ernesto y GODOY ORELLANA, Milton, Construcción estatal, orden oligárquico y respuestas sociales, Argentina y Chile, 1840-1930, Universidad Nacional de General Sarmiento, Buenos Aires, 2010; HARAMBOUR, Alberto, "Borderland Sovereignties. Postcolonial Colonialism and State Making in Patagonia. (Argentina and Chile, 1840s-1922)", Tesis doctoral, Stony Brook, 2012.

10 Recientes estudios sobre el sistema bracero han comenzado ha explorar el impacto de este sistema de migración y contratación laboral en diferentes estados de México. Ver por ejemplo: ALANIS, Fernando, "La contratación de braceros en SLP y el gobierno del estado (1944)," en ALANIS, Fernando, Emigración de San Luis de Potosí a Estados Unidos: Pasado y presente, El Colegio de San Luis, San Luis, 2001. Un caso similar analiza HAMOVITCH, Cindy, No Man's Land: Jamaican Guestworkers in America and the Global History of Deportable Labor, Princeton University Press, Princeton, 2011.

11 Sobre la relación entre Imperialismo norteamericano y trabajo en América Latina véase especialmente: GREEN, July, The Canal Builders: Making America's Empire at the Panama Canal, Penguin, Londres, 2009; LIPMAN, Jana Guantánamo: A Working-Class History Between Empire and Revolution, California University Press, Berkeley, 2008; TÍNKER-SALAS, Miguel, The Enduring Legacy: Oil, Culture, and Society in Venezuela, Duke University Press, Durham, 2009. 
tipología a lo largo del continente. Nos interesa fundamentalmente levantar preguntas sobre cómo podemos hacer una historia transnacional del trabajo desde la "periferia" y cómo establecer comparaciones entre América Latina y Estados Unidos sin desconocer las profundas y desiguales relaciones de poder. En una primera parte, se analizará el desarrollo del paternalismo industrial en América Latina, señalándose algunas de sus trasnformaciones y características centrales, para luego abocarnos específicamente al caso de las ciudades empresas y la industria minera.

\section{Industrialización y Paternalismo en América Latina}

Los procesos de industrialización y modernización de la producción no sólo requirieron innovaciones tecnológicas, nuevas políticas económicas y una estructura financiera moderna, sino que además, una profunda transformación de la mano de obra. Esta transición a un régimen de trabajo industrial significó adaptarse e incorporar nuevos hábitos, disciplinas y normas, desarrollar otro tipo de habilidades y técnicas como así también aprender a responder a la relación salarial y las demandas del mercado. Tal como lo ha señalado la riquísima historiografía sobre el proceso de proletarización, esta transición significó también el desarrollo de nuevas identidades, ideologías, conciencias y prácticas políticas ${ }^{12}$. Frente a los desafíos de crear y controlar una mano de obra moderna, los empresarios industriales comenzaron a discutir y diseñar distintas estrategias de "ingeniería laboral", las cuales, sin embargo, nunca sustituyeron completemante formas tradicionales de represión. En el mundo industrial del siglo XIX, surgió la imagen del patrón o patriarca benevolente y autoritario que buscaba proteger, educar y modernizar a su subordinada "familia" de empleados. Durante las primeras décadas del siglo XX, este patrón paternalista comenzó a ser remplazado por Departamentos de Bienestar y los beneficios que otorgaba la empresa abandonaron su carácter de "premios personales" y pasaron a ser parte de un atractivo paquete de beneficios sociales ${ }^{13}$. Estos cambios en las ideas y prácticas patronales fueron parte de una transformación más amplia de como se pensaba y diseñaba la producción industrial a lo largo del siglo XX.

El paternalismo industrial ejerció una profunda influencia en los Estados Unidos y en otros países industrializados. Si la industria textil fue un ejemplo del paternalismo clásico del siglo XIX, a comienzos del siglo XX y en respuesta a los profundos cambios políticos, laborales, tecnológicos y económicos surgió en la nueva industria de producción masiva un nuevo modelo de paternalismo industrial. El llamado welfare capitalism (paternalismo

12 Entre muchos otros, véase por ejemplo los extensos trabajos de Julio Pinto Vallejos y Gabriel Salazar para el caso de Chile, Ricardo Salvatore para Argentina, William French para México y Heraclio Bonilla en Perú.

13 Una interesante discusión teórica nos ofrece el libro de SENNET, Richard, Authority, Norton, Nueva York, 1993.

\section{8}


industrial) o welfare work (trabajo bienestar), nos señala Andrea Tone, alcanzó popularidad en Estados Unidos durante las primeras dos décadas del siglo XX, cuando "Los empleadores usaron welfare work para reorganizar antiguas prácticas de control laboral y así hacer frente a las exigencias sociales y políticas de esa época" ${ }^{14}$. Esto significó que esta nueva variante de welfare capitalism en los Estados Unidos incorporara tres elementos claves: (1) antiestatismo como respuesta a la creciente intervención y regulación del Estado; (2) políticas de género como reacción a la creciente incorporación de la mujer a nuevos espacios de trabajo; y (3) énfasis en la productividad y eficiencia para hacer frente a un mercado cada vez más competitivo. La empresa automotriz Ford se levantó como modelo de empresa benefactora, desarrollando un complejo sistema de beneficios e incentivos que se extendían desde la fábrica hasta la vida familiar y privada del trabajador. Para Henry Ford, el sistema Fordista sería la base de la expansión industrial ya que no sólo consolidaría una mano de obra eficiente y moderna sino además aumentaría el poder adquisitivo de los propios trabajadores quienes se convertirían en consumidores de la creciente oferta de productos industriales ${ }^{15}$.

¿Cómo este paternalismo industrial y las experiencias norteamericanas influyeron en el desarrollo industrial de América Latina? ¿Es posible hablar del traspaso de ideas y prácticas de bienestar desde Estados Unidos al resto del continente? Y, si este fuese el caso ¿Cómo circularon y eventualmente se adaptaron estas ideas a lo largo del continente? ¿Cómo periodizamos la historia del paternalismo industrial en América Latina y, hasta qué punto, esta periodización coincide con la periodización que han realizado historiadores norteamericanos? ¿Cuál fue el papel que jugaron las empresas extranjeras en la divulgación de estas ideas y prácticas? En otras palabras, y desde una perspectiva más amplia, ¿Podemos utilizar conceptos, ideas e interpretaciones concebidos para el mundo industrial desarrollado en un contexto de desarrollo industrial tardío y periférico?

A comienzos del siglo XX, los procesos de industrialización, modernización y expansión capitalista en América Latina motivaron la discusión e implementación de nuevas prácticas, tanto estatales como privadas, de control laboral. Así en algunas de las industrias más grandes, los empresarios industriales comenzaron a incorporar una serie de beneficios sociales que buscaban complementar el salario. Se diseñaron y construyeron viviendas, escuelas y otros servicios sociales y urbanos tanto para el trabajador como para su familia. Este discurso era parte de una preocupación mayor por incorporar nuevas tecnologías y desarrollar, expandir y racionalizar la producción industrial. De esta forma la ingeniería de la producción llegó de la mano de la llamada "ingeniería social" y -en muchos casos- de la "ingeniería racial".

¿Cuáles fueron las características de este paternalismo industrial Latinoamericano?

14 TONE, Andrea, The Business of Benevolence: Industrial Paternalism in Progressive America, Cornell University Press, Ithaca, 1997.

15 MEYER, Stephen, The Five-Dollar Day: Labor Management and Social Control in the Ford Motor Company, 1908-1921, State University of New York, Albany, Nueva York, 1981. 
$\mathrm{Al}$ otorgar estos beneficios (los cuales eran muchas veces superiores a los que recibían o tenían acceso otros sectores de trabajadores), la empresa buscaba ejercer y moldear, por ejemplo, las costumbres, las relaciones de género y de familia, los hábitos alimenticios y de consumo de sus empleados y obreros, creando además un complejo entrenzado entre los espacios propiamente de trabajo y aquellos de vida familiar y social. Así, donde empezaba o terminaba el ámbito de la empresa o cuales eran los límites entre lo laboral y lo estrictamente privado nunca estaba totalmente claro para estos trabajadores. El tema de la lealtad y el vínculo entre empresarios y trabajadores pareciera haber sido también una preocupación central. Por ejemplo, en el caso del Ferrocarril Central Argentino, nos explica Laura Badaloni, "el despliegue de acciones de rasgo paternalistas cuya meta era el involucramiento de los trabajadores con los intereses de la compañía"16. Para Guillermo Guajardo, las prácticas paternalistas en las empresas ferroviarias Mexicanas respondían también a la necesidad de capacitar a los trabajadores e imponer una disciplina industrial ${ }^{17}$. Una situación similar nos describe Paulo Fontes en el caso de la industria paulista. En el ABC paulista, los beneficios no salariales y las prácticas paternalistas eran formas de "transformar ex trabajadores rurales en operarios fabriles" ${ }^{18}$. En empresas con gran número de mujeres, como fue el caso de la industria textil, el paternalismo industrial también surgió como una forma de control de la mujer y la familia obrera ${ }^{19}$.

Un claro ejemplo de estas prácticas paternalistas son las llamadas comunidades fábricas de las primeras décadas del siglo XX. En la Vila Operária da Boa Viagem construida por el empresario Luiz Tarquino en la ciudad de Salvador de Bahía o en las comunidades de Votorantim (1918) y Vila Operaria Maria Zélia (1917) en el Estado de São Paulo, la construcción de viviendas y la entrega de servicios sociales eran consideradas fundamentales para el éxito de la producción textil. En estas comunidades planificadas, el empleador

16 BADALONI, Laura, "La familia ferroviaria a principios del siglo XX Bienestar y lealtades de hierro en el Ferrocarril Central Argentino", en DICÓSIMO, Daniel y SIMONASSI, Silvia (compiladores) Trabajadores y empresarios en la Argentina del siglo XX: Indagaciones desde la historia social, Prohistoria ediciones, Rosario, 2011.

17 GUAJARDO SOTO, Guillermo, Trabajo y tecnología en los ferrocarriles de México: Una visión histórica, 1850-1950, Consejo Nacional para la Cultura y las Artes, Ciudad de México, 2010.

18 FONTES, Paulo, Um nordeste em São Paulo: trabalhadores migrantes em São Miguel Paulista (1945-66), Editora FGV, Rio de Janeiro, 2008.

19 Sobre políticas de género y paternalismo industrial ver por ejemplo: FARNSWORTH-ALVEAR, Ann, Dulcinea in the Factory: Myths, Morals, Men and Women in Colombia's Industrial Experiment, 1905-1960, Duke University Press, Durham, 2000; HUTCHISON, Elizabeth, Labors Appropriate to Their Sex: Gender, Labor, and Politics in Urban Chile, 1900-1930, Duke University Press, Durham, 2001; LOBATO, Mirta, La vida en las fábricas. Trabajo, protesta y política en una comunidad obrera, Berisso (1904-1970), Prometeo, Buenos Aires, 2001; PORTER, Susie, Working Women of Mexico City: Public Discourse and Material Conditions, 1871-1930, University of Arizona Press, Tucson, 2003. 
aspiraba crear un trabajador sano, limpio, bien alimentado, sin preocupaciones y aislado de las "malas" influencias sociales, biológicas y políticas. Estas nuevas ciudades y barrios ofrecían un nuevo modelo de orden, modernidad y progreso. Las habitaciones homogéneas, el cuidadoso trazado de sus calles y la construcción de espacios controlados de esparcimiento buscaban remplazar el conventillo hacinado, los pasajes y callejones sucios, la taberna y el prostíbulo, lugares donde el alcoholismo, la enfermedad y la promiscuidad amenazaban con degenerar a la población trabajadora ${ }^{20}$.

La influencia del paternalismo industrial en América Latina fue desigual y heterogénea. Tal como lo señala Barbara Weinstein para el caso de Brasil, existía una brecha entre el discurso industrial y la práctica. En parte, esto se debió a las características propias del proceso de industrialización en el continente. Por un lado, la combinación de algunos pocos complejos industriales modelos y a gran escala y una gran mayoría de pequeñas empresas y talleres significó que gran parte del sector industrial no estaba en condiciones de desarrollar extensos sistemas de bienestar. En Brasil, por ejemplo, en la década de 1920, las condiciones de producción, la abundancia y bajo costo de la mano de obra y la poca necesidad de mejorar la productividad desalentaban invertir en este tipo de programas ${ }^{21}$. Es decir, aunque influidos por un discurso modernizador e industrializador, muchos empresarios creían que para innovar el proceso productivo, racionalizar la producción o introducir nueva tecnología era necesario transformar los hábitos del trabajador, la realidad cotidiana de la fábrica tendía a decir otra cosa.

A pesar de estas limitantes, observamos que en las fábricas, explotaciones mineras y establecimientos agro-industriales se comenzaron a implementar con mayor regularidad prácticas y programas de bienestar a partir de la década de 1920, extendiéndose sobre todo a partir de la década de 1940 a medida que avanzaba el proceso de industrialización y substitución de importaciones. Empresarios, elites locales y el Estado celebraron la construcción de villas obreras y la implementación de sistemas paternalistas como símbolos del progreso industrializador y modernidad a que aspiraban. A fines de la década de 1920, la Compañía Carbonifera de Lota y Coronel organizó uno de los primeros servicios sociales-industriales modernos en Chile. Para ello, la empresa contrató los servicios de una asistente social, quien se hizo cargo de supervisar la escuela, los servicios materno-infantiles y de visitar las familias obreras y distribuir los beneficios sociales que otorgaba la empresa. Asimismo, tal como

20 Véase por ejemplos los trabajos de ALTERMAN BLAY, Eva, Eu nao tehno onde morar. Vilas operarias na cidade de Sao Paulo, Livraria Nobel, Sao Paulo, 1985; LEITE LOPES, José Sergio, "Fábrica e Vila Operária: Considerações sobre uma forma de servidão burguesa", en: Mudança social no Nordeste: A reprodução da subordinação, Paz e Terra, Rio de Janeiro, 1979; NEIBURG, Federico, Fábrica y villa obrera: historia social y antropología de los obreros del cemento, Centro Editor de América Latina, Buenos Aires, 1988.

21 WEINSTEIN, Barbara, For Social Peace in Brazil: Industrialists and the Re-Making of the Working Class in Sao Paulo, 1920-1964, Chapel Hill, North Carolina University Press, 1997. 
lo hacía Ford Motors en Estados Unidos, esta empresa carbonífera estableció un riguroso sistema de premios mensuales para los trabajadores y las familias modelos. A través de la gestión de la visitadora social y los beneficios sociales, nos señala María Angélica Illanes, la empresa entraba "al interior de la intimidad de la habitación obrera, insertando al trabajador en el flujo continuo de la producción por la vía de la domesticidad". ${ }^{22}$

El paternalismo industrial en América Latina no surgió necesariamente en oposición al Estado, como nos sugiere Andrea Tone para el caso de los Estados Unidos; sino, por el contrario, muchas veces complementó los servicios públicos y mantuvo una estrecha relación con las instituciones del Estado. En cierta medida, es importante tomar en cuenta que la divulgación de las prácticas paternalistas coincidió en América Latina con la expansión de la legislación social, el paternalismo del Estado y, a partir de fines de mediados de la década del treinta, con los proyectos populistas. ${ }^{23}$ Asimismo, las empresas públicas y estatales que fueron surgiendo a partir de la década de 1940 y se constituyeron en pilares del proceso de substitución de importaciones de las décadas siguientes consolidaron el paternalismo industrial como eje central de las relaciones entre capital y trabajo. ${ }^{24}$

A pesar de esta compleja trama de beneficios sociales, tal como lo señala Peter Winn en su ya clásico estudio de la industria textil en Chile a lo largo del siglo XX, el paternalismo industrial nunca eliminó las prácticas patronales más coercitivas, desarrollándose muchas veces una especie de "paternalismo represivo". ${ }^{25}$ Además la incorporación del Taylorismo a mediados del siglo XX, como instrumento para reorganizar el proceso productivo fabril y ejercer mayor control sobre los espacios de trabajo, fue erosionando las formas de control patronal. De esta forma, tal como se examina al final de este artículo, el paternalismo industrial no logró impedir la organización de comunidades obreras autónomas, políticas y empoderadas.

22 ILLANES, María Angélica, "Ella en Lota-Coronel: Poder y domesticación en el primer servicio social industrial en América Latina", Mapocho, Santiago, 2001, pp. 141-148.

23 Véase por ejemplo la sugerente discusión de Paulo Drinot sobre el caso Peruano, especialmente su capítulo sobre habitaciones obreras. DRINOT, Paulo, The Allure of Labor: Workers, Race and the Making of the Peruvian State, Durham, Duke University Press, 2011.

24 Ver por ejemplo los casos descritos por: DINIUS, Oliver, Brazil's Steel City: Developmentalism, Strategic Power, and Industrial Relations in Volta Redonda, 1941-1964, Stanford University Press, Palo Alto, 2010; NOVELO, Victoria y ARTEAGA, Augusto, La industria de los Magueyales: trabajo y sindicato en Ciudad Sahagún, Editorial Nueva Imagen, México, 1979.

25 WINN, Peter, “El Taylorismo y la gran huelga de Yarur de 1962”, en Proposiciones, Chile, 1990, No 19, pp. 202-222 y Los tejedores de la revolución: Los trabajadores de Yarur y la vía Chilena al socialismo, LOM, Santiago, 2004. 


\section{Los campamentos mineros: ¿paternalismo industrial ejemplar?}

La influencia del paternalismo industrial se nota con especial claridad en el caso de la expansión de la minería. A partir de fines del siglo XIX, la industria minera comenzó una profunda reorganización empresarial y cambio tecnológico. El desarrollo de un sector minero moderno, industrial y capitalista requirió además de la expansión e integración de sus servicios tales como las plantas hidroeléctricas, ferrocarriles y sistemas de abastecimiento de insumos. Del mismo modo, estas nuevas empresas mineras se enfrentaron al desafío de como garantizar una fuerza de trabajo estable, contar con personal técnico capacitado y controlar el conflicto social en condiciones, muchas veces, de gran aislamiento geográfico ${ }^{26}$. $\mathrm{Al}$ igual que los empresarios industriales en las ciudades, las nuevas empresas mineras se inspiraron en el paternalismo industrial y construyeron comunidades laborales y campamentos "modelos".

El campamento "paternalista" se distinguió de los antiguos poblados mineros por su cuidadoso diseño y trazado, la existencia de departamentos de bienestar, el control y presencia hegemónica de la empresa y la entrega no solo de lo mínimo para la subsistencia sino de un paquete de atractivos servicios sociales. Si los antiguos poblados mineros eran un lugar de crecimiento desregulado y caótico y sus habitantes, por lo general, peones jóvenes y solteros; el nuevo campamento construido por la empresa buscaba atraer trabajadores casados, técnicos y familias. Así, estas pequeñas ciudades planificadas aspiraban convertirse en polos de atracción, retención, control y proletarización de una mano de obra de origen obligadamente migrante. El aislamiento geográfico y el carácter migrante de la mano de obra otorgaron al campamento minero y petrolero elementos únicos, muchas veces extremos.

Nicolás Cárdenas, por ejemplo, señala que en gran parte de la minería industrial mexicana de comienzos del siglo XX se construyeron campamentos modelos que contaban con casas, hospitales, puestos de socorro, boticas y tiendas de rayas. A diferencia de las tradicionales tiendas de rayas que explotaban y endeudaban al trabajador, en las nuevas empresas de El Boleo, Moctezuma Copper, Nacozari y Cananea, se vendían productos a precios más bajos como una forma de atraer obreros. Para Cárdenas estos servicios no representarían una forma clásica de paternalismo, sino "un conjunto de medidas necesarias dentro de la racionalidad de la empresa capitalista. La habitación, los hospitales, el alumbrado público, el agua potable, las escuelas, y las tiendas, tenían el propósito de cuidar una fuerza de trabajo específica, en muchas partes escasa y también inflamable. Por eso mismo buscaban a

26 Para una visión general de la historia de la minería en América Latina, BROWN, Kendall, $A$ History of Mining in Latin America From the Colonial Era to the Present, University of New Mexico Press, Albuquerque, 2012. 
la vez inculcarle hábitos adecuados dentro de grandes unidades industriales, para así evitar protestas mayores que frenaran su eficaz funcionamiento" 27 .

No todas las empresas mineras construyeron campamentos mineros completos, y muchas de ellas utilizaron o adaptaron antiguos poblados cercanos a las ciudades. Por ejemplo, en el caso de la minera andina peruana, mientras Cerro de Pasco se instaló en un antiguo poblado minero y se limitó a expandir la infraestructura urbana existente, en La Oroya, la compañía construyó una cuidad empresa, "una nueva aglomeración formada en un paraje donde no existían antecedentes de centros poblados de envergadura" ${ }^{28}$. Pero en ambos casos, la empresa ejercía un estricto control sobre los espacios de vida de los obreros y empleados, haciendo sentir su presencia e influencia más allá de los espacios de trabajo tradicional.

La expansión de la gran minería en América Latina estuvo íntimamente ligada a la expansión del capital extranjero y muchos de estos campamentos se volvieron emblemáticos sitios de propiedad extranjera ${ }^{29}$. Estos se caracterizaron por la extrema división y segregación entre una mano de obra nacional/local y un empresariado extranjero en el espacio urbano y en los sistemas de pago (moneda local para los trabajadores y dólar para el extranjero), que se sustentaba en un discurso de superioridad racial y cultural. La segregación del espacio urbano, la presencia de administradores y profesionales norteamericanos (y algunos europeos), el uso del inglés en muchas de las comunicaciones internas de la empresa y la utilización de maquinaria y herramientas extranjeras reforzaban la imagen de un enclave norteamericano. En el caso del yacimiento de cobre El Teniente en Chile, el historiador Thomas Klubock, nos describe un campamento en el cual la presencia norteamericana reforzaba las tradicionales divisiones de clase. ${ }^{30}$

Esta enorme capacidad tecnológica, económica y social de la empresa extranjera tuvo un fuerte impacto en algunos sectores de las élites locales, quienes vieron en ellos un modelo de modernización tanto productiva como social. Por ejemplo en el caso de Chile, las páginas de las principales revistas empresariales (Sociedad Nacional de Minería, Sociedad de Ingenieros, o Sociedad de Fomento Fabril) contienen abundantes referencias al modelo norteamericano de organización empresarial que se implementaba en la industria del cobre,

27 CÁRDENAS GARCÍA, Nicolás, Empresas y trabajadores en la gran minería Mexicana 19001929. Instituto Nacional de Estudios Históricos de la Revolución Mexicana, México, 1998.p.135.

28 VEGA-CENTENO, Pablo, "Los efectos urbanos de la minería en el Perú: del modelo de Cerro de Pasco y La Oroya al de Cajamarca", en Apuntes, Lima, 2011, № 68, pp. 109-136

29 O'BRIEN, Thomas, Revolutionary Mission: American Enterprise in Latin America, 1900-1945, Cambridge University Press, Cambridge, 1999.

30 KLUBOCK, Thomas M., Contested Communities: Class, Gender, and Politics in El Teniente's Copper Mine, 1904-1951, Duke University Press, Durham: 1998; "Nationalism, Race, and the Politics of Imperialism: Workers and North American Capital in the Chilean Copper Industry", en JOSEPH, Gilbert, Reclaiming the Political in Latin American History: Essays from the North, Duke University Press, Durham, 2001.

\section{4}


sugiriendo el traspaso de algunas prácticas norteamericanas al medio local. Así, en 1919 el periódico La Verdad (Chañaral), resaltaba los logros alcanzados por ingenieros norteamericanos en la mina de Potrerillos. Estos hombres trabajadores, eficientes, disciplinados, austeros, y bien educados habrían construido una obra maestra de ingeniería y modernidad. Los "hijos de la Gran República", como La Verdad llamaba cariñosamente a los extranjeros de Potrerillos, habían salvado, exitosamente, los obstáculos geográficos y técnicos debido a "su alta capacidad empresarial" y su "sólida educación." ${ }^{11}$ En los próximos años, periódicos y revistas técnicas enfatizaron la labor de las compañías norteamericanas, subrayando -como una revista local explicaba en 1932- su "rigurosa base científica". ${ }^{32}$

Algunos historiadores han destacado el impacto de la presencia extranjera en los hábitos sociales, prácticas culturales e ideas políticas de los trabajadores. En estos espacios surgió muchas veces un temprano nacionalismo que se constituyó en elemento central del discurso de las organizaciones sindicales y políticas. ${ }^{33}$ En las minas de cobre de Canenea, por ejemplo, el historiador Juan Luis Sariego nos describe la profunda discriminación racial que existía en las enclaves mineros del norte de México. En estos enclaves, Sariego señala, el conflicto obrero se desarrolló con profunda intensidad, un "conflicto sin intermediarios polarizado entre empresarios y trabajadores cuya expresión más recurrente es la revuelta anarquista y su móvil, la defensa obrera del nacionalismo" ${ }^{34}$ El nacionalismo y la revuelta, sin embargo, no fueron las únicas respuestas al proyecto paternalista industrial extranjero. Tal como lo ha demostrado Miguel Tínker-Salas en su estudio sobre los campamentos petroleros en Venezuela, lo foráneo también ejerció una influencia cultural importante sobre la mano de obra, creando muchas veces comunidades híbridas que incorporaban algunos aspectos de la cultura norteamericana (vocablos y expresiones lingüísticas, deportes, artículos de consumo, celebraciones, etc.). ${ }^{35}$

Los historiadores han debatido largamente el impacto del paternalismo industrial, las ciudades empresas y de los beneficios otorgados por las empresas sobre los trabajadores y sus familias. ¿Lograron éstos disciplinar, fijar geográficamente o influir sobre la mano de

31 La Verdad, Chañaral, 26/03/1919.

32 Carlos Ramírez and Graciela Navarrete de Ramírez, "Potrerillos," Publicación extraordinaria Atacama, mayo 1932, pp. 12-27.

33 Charles Bergquist ahonda en las experiencias comunes de los trabajadores del sector exportador. BERGQUIST, Charles, Labor in Latin America: Comparative Essays in Chile, Argentina, Venezuela and Colombia, Palo Alto, Stanford University Press, 1996.

34 Juan Luis Sariego ha estudiado extensamente el caso de Cananea en México. SARIEGO, Juan Luis, Enclaves y minerales en el norte de México: Historia social de los mineros de Cananea y Nueva Rosita, 1900-1970, Centro de Investigaciones y Estudios Superiores en Antropología Social, México, 1988, p. 35.

35 TÍNKER-SALAS, The Enduring Legacy..., Op. Cit. 
obra? ¿Creó el paternalismo una mano de obra dócil y obediente? ¿Fueron las viviendas otorgadas por la empresa o la intervención en los espacios de la vida privada formas efectivas de desmovilización y control patronal? José Sergio Leite Lopes en su ya clásico estudio sobre los obreros del azúcar en el Brasil se refiere a las viviendas de la usina, la entrega de recursos y beneficios diarios (ej.: leña, mantenimiento, huertos) y la organización del tiempo libre como formas de sometimiento. Para acceder a estos beneficios, los obreros se relacionan con los empleados de la empresa, quienes actuaban como suerte de puente entre los obreros y la administración. "Los trabajadores residentes en el barrio obrero", nos señala Leite Lopez, "pasan entonces a depender necesariamente de todo un tejido de 'favores' por parte de los empleados. Esa trama de 'favores' que se manifiesta en su esfera doméstica completa la trama de 'favores' de la esfera del trabajo"36. De esta forma, la empresa azucarera mantenía un estricto control sobre todos los aspectos de la vida y el trabajo obrero, los cuales, para Leite Lopes, creaban condiciones similares de control a las instituciones de asilo. Sin embargo, si estos favores lograron muchas veces dividir a los obreros, los constantes esfuerzos de los ingenios azucareros por reducir gastos o "expropiar" parte de los beneficios reforzaban, en tiempos de crisis, los vínculos entre los trabajadores y su oposición a la empresa.

El caso de los trabajadores del azúcar descrito por Leite Lopes nos sugiere una forma distinta de ver los beneficios de la empresa paternalista. Para los obreros de la azúcar, la "expropiación" de los beneficios era interpretada como "una burla de la administración al "contrato' de trabajo informal" ${ }^{37}$. En otras actividades económicas, quizás menos opresivas que la industria azucarera, los esfuerzos de la empresa por reducir, controlar o condicionar la entrega de beneficios era interpretada por los trabajadores y sus organizaciones sindicales como un ataque directo a lo que eran considerados derechos. En otras palabras se observa como los beneficios del paternalismo pasaron de ser beneficios otorgados por la empresa a convertirse en derechos y conquistas laborales.

El caso de la Gran Minería del Cobre ${ }^{38}$ en Chile nos ofrece un claro ejemplo de esta transición y de los límites del paternalismo industrial y las ciudades empresas. Construidos en la década de 1920, los campamentos de la GMC se convirtieron rápidamente en ejemplos de eficientes ciudades empresas. Junto a la vivienda, los trabajadores tenían acceso a una amplia gama de beneficios sociales, urbanos y recreativos que creaban condiciones de dependencia que iban más allá del trabajo. ${ }^{39}$ Sin embargo, la presencia de la empresa en la

36 LEITE LOPES, José Sergio, El vapor del diablo. El trabajo de los obreros del azúcar, Antropofagia, Buenos Aires, 2011, p. 267.

37 Ídem, p. 278.

38 En adelante GMC.

39 Sobre la historia laboral del cobre ver: KLUBOCK, Thomas, Contested Communities: Class, gender, and Politics in Chile's El Teniente Copper Mine, 1904-1951, Duke University Press, Durham, 1998; 
esfera privada y en la vida social y cotidiana de los trabajadores así como la intersección de los espacios de trabajo y no-trabajo entrelazaron las demandas laborales y aquellas de carácter urbano o comunitario. A la larga, este entretejido contribuyó a unificar y politizar a los trabajadores y sus familias en torno a un "enemigo" común: la empresa. Si en una ciudad, el problema de la distribución de alimentos, por ejemplo, habría sido un conflicto entre las autoridades locales y la población, en los campamentos del cobre era un conflicto entre capital y trabajo ${ }^{40}$.

La administración y organización de los campamentos del cobre, entonces, provocó serios y continuos conflictos sociales, desde manifestaciones espontáneas de los residentes (generalmente lideradas por las esposas y dueñas de casa) a reclamos formales de los sindicatos, huelgas generales y paros sectoriales. Si la empresa había buscado establecer una presencia hegemónica tanto en los espacios de trabajo como de la vida cotidiana, las organizaciones de trabajadores incorporaron rápidamente los espacios de no-trabajo al ámbito de las relaciones laborales, exigiendo de la empresa desde el mejoramiento de las condiciones habitacionales a la ampliación de los beneficios sociales. La intersección de los espacios de vida y trabajo lejos de desmovilizar a los trabajadores, empoderaron las organización sindicales, cuyo radio de acción se extendía desde los problemas estrictamente relacionados al trabajo, a lo comunitario, urbano y cotidiana, involucrando a las mujeres y las familias, generalmente ausentes de las luchas sindicales tradicionales. En otras instancias, los propios trabajadores desarrollaron instituciones alternativas, reclamando espacios autónomos de socialización, consumo o politización.

Los aspectos coercitivos y controladores del paternalismo industrial naturalmente fueron a largo plazo resquebrajando el sistema. El paternalismo industrial buscaba controlar no empoderar al trabajador, y aunque algunos trabajadores, tal como lo señala Peter Winn, en algún momento fueron cooptados por las relaciones y beneficios paternalistas (a los que Winn denomina los trabajadores "apatronados"), eventualmente el sistema tendió a ser resentido y cuestionado. Si por un lado estaban los beneficios sociales, por otro lado estaban los representantes del Departamento de Bienestar que inspeccionaban semanalmente las casas para constatar que sus habitantes se ajustaban al reglamento. A la larga, el paternalismo industrial, la ciudad empresa y el campamento minero modelo fracasaron como herramientas de control laboral.

FINN, Janet L., Tracing the Veins: Of Copper, Culture and Community from Butte to Chuquicamata, University of California Press, Berkeley, 1998; VERGARA, Ángela, Copper Workers, International Business and Domestic Politics in Cold War Chile, Pennsylvania State University Press, University Park, 2008.

40 La problemática del consumo se dio fundamentalmente en torno a los precios y distribución de los productos de primera necesidad y al papel desempeñado por las tiendas de raya o pulperías. VERGARA, Ángela, "Precios y raciones: la Anaconda Copper Company en Chile entre 1932 y 1958", Investigaciones de Historia Económica, España, octubre 2012, V.VIII, No III, pp. 135-143. 


\title{
Conclusiones
}

El paternalismo industrial cumplió un rol fundamental en el proceso de modernización e industrialización de América Latina. Inspirados muchas veces en modelos extranjeros y en las prácticas del paternalismo industrial en boga en esos años, empresarios locales y extranjeros construyeron viviendas y establecieron complejos sistemas de bienestar social. La nueva empresa moderna consideraba la inversión e intervención en la vida cotidiana y social de los trabajadores -en otras palabras la ingeniería social- absolutamente esencial para el éxito del nuevo modelo industrial, el cual dependía de una fuerza de trabajo estable, sana y dócil. Con ello, el empresariado se unió a los esfuerzos de médicos, higienistas, educadores, asistentes sociales y funcionarios públicos que buscaban limpiar y erradicar los vicios del bajo pueblo, creando la base de una población moderna que pudiese contribuir al progreso social, político y económico del país. A través del paternalismo se buscó inculcar nociones de trabajo capitalista que iban desde los sistemas de turnos y horarios, la productividad, el compromiso con la producción hasta la transformación de los hábitos personales de los trabajadores y sus familias (nutrición, control del alcohol, estructura familiar). Sin embargo, sus aspectos coercitivos y controladores eventualmente erosionaron el sistema, y en la medida en que los trabajadores transformaron los beneficios sociales paternalistas en derechos y conquistas, el empresariado cuestionó su utilidad y tanto las ciudades empresas como los departamentos de bienestar fueron perdiendo vigencia.

La historia de las múltiples y diversas comunidades laborales creadas por el empresariado a lo largo del continente nos ofrece oportunidades únicas para comprender la historia de los trabajadores y los empresarios en América Latina. No solo nos sugiere las semejanzas en las formas de organizar la industria (tanto productivamente como en sus aspectos socio-laborales) sino además en las respuestas de los propios trabajadores. A través de la organización sindical, el conflicto, la alianza con el Estado, la politización o la creación de instituciones alternativas (tales como las cooperativas, periódicos, clubes), los trabajadores se fueron empoderando y transformando los proyectos empresariales de corte paternalista.

\author{
Recibido: 04/06/2013
}

Aceptado: 26/09/2013 